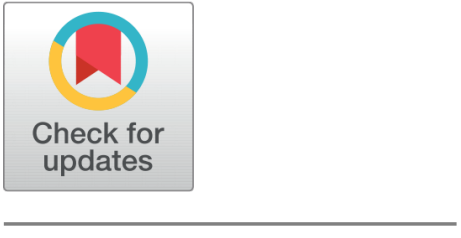

OPEN ACCESS

Received: 09.04.2021

Accepted: 07.05.2021

Published: 21.05 .2021

Citation: Vatamanu D, Iftode C, Miclaus S (2021) Microstrip patch and Sierpinski fractal antennas: simulations and experimental characterization for vital signs detection with Doppler radar technique. Indian Journal of Science and Technology 14(18): 1477-1487. https://doi.org/ 10.17485/IJST/v14i18.614

* Corresponding author.

Tel: +40-269432990

simo.miclaus@gmail.com

Funding: None

Competing Interests: None

Copyright: (c) 2021 Vatamanu et al. This is an open access article distributed under the terms of the Creative Commons Attribution License, which permits unrestricted use, distribution, and reproduction in any medium, provided the original author and source are credited.

Published By Indian Society for Education and Environment (iSee)

ISSN

Print: 0974-6846

Electronic: 0974-5645

\section{Microstrip patch and Sierpinski fractal antennas: simulations and experimental characterization for vital signs detection with Doppler radar technique}

\author{
David Vatamanu ${ }^{1,2}$, Cora Iftode ${ }^{3}$, Simona Miclaus ${ }^{2 *}$ \\ 1 Faculty of Engineering, "Lucian Blaga” University, 1-3 Emil Cioran St., Sibiu, Romania \\ 2 Communication and IT Department, "Nicolae Balcescu" Land Forces Academy, 3-5 \\ Revolutiei St., Sibiu, Romania. Tel.: +40-269432990 \\ 3 Measurements and Optical Electronics Department, Politehnica University of Timisoara, \\ Romania
}

\section{Abstract}

Objectives: To fabricate planar models of antennas with resonance frequencies in the ultrahigh frequency band that allow short-range detection of respiration and heartbeat by a simple continuous wave Doppler radar system. Methods: Models of antennas were created in CST Studio software and the main parameters were computed. Then, antennas were fabricated at a PCB prototyping machine and were experimentally characterized in an anechoic chamber. Total efficiency and radiation patterns indicated the best working frequencies of $2.12 \mathrm{GHz}$ and $8.82 \mathrm{GHz}$ respectively, to test the human vital signs detection with a continuous-wave Doppler radar technique in direct visibility conditions. Findings: The patch antenna at $2.12 \mathrm{GHz}$ had a maximum gain of $3.15 \mathrm{dBi}$ and total efficiency of $43 \%$ while the Sierpinski antenna at $8.82 \mathrm{GHz}$ had a maximum gain of $5.5 \mathrm{dBi}$ and total efficiency of $65 \%$. At incident power densities on the human subject's chest of $4.5 \times 10^{-4} \mathrm{~mW} / \mathrm{m}^{2}$ and of $2.6 \times 10^{-2} \mathrm{~mW} / \mathrm{m}^{2}$ respectively, the Doppler radar system based on these antennas offered precise responses. Practically, it was possible to extract both the heartbeat rate and the respiration rate, by simply applying the classical FFT algorithm on a time-series phase data of the transmission coefficient recorded during $30 \mathrm{sec}-$ onds, when the set-up was composed of just the antennas and a vector network analyzer. Novelty: Increased detection accuracy was obtained due to careful characterization of the antennas parameters with no need of special processing algorithms.

Keywords: Sierpinski Antenna; Patch Antenna; CW Doppler Radar;

Respiration Rate; Heartbeat Rate

\section{Introduction}

The development of antennas for various applications is a branch of continuous development. Choosing one certain application has implications on which parameters 
are of the greatest importance for designing and characterizing an antenna. The present approach aimed at a comprehensive characterization of two in-house fabricated antennas using the printed circuit board (PCB) technique, to establish their proper use for vital signs detection based on continuous wave (CW) Doppler radar principle. Practically, two types of PCB antennas were designed and fabricated, analyzed and used for preliminary tests of detecting respiration and heartbeat rhythms of a person.

Microstrip patch antennas are consisting of a pair of good conducting layers separated by a dielectric substrate. The upper conducting layer is the radiating/active part of the antenna, while the bottom layer is the ground plane. Often, copper is used as a conductor. The active face may have various geometric shapes while the antenna's dimensions are expressed in terms of mathematical relations ${ }^{(1,2)}$. The performances are highly dependent on the dimensions but also on the permittivity of the dielectric substrate. Generally, the patch antenna has narrow bandwidths. During the patch design, one needs to connect the active surface with the ground plane. Various techniques are used, influencing also the performances. Having lots of advantages supplemented by the possible reconfigurability, patch antennas are present today in laptops (for Wi-Fi, GPS, Bluetooth connections, etc.), mobile phones, WiMax networks, MIMO systems, cognitive radios, satellite communications, but also in radars and body-centric communications ${ }^{(3,4)}$.

Fractal antennas are formed by multilevel and space-filling conductive curves, repeating a motif over two or more scale sizes, called iterations. The main characteristic is their highest compactness. They feature multi- or wide band functioning, the fact that enables their uses in cellular, microwave- or body-centric communications. Miniaturization, band increase, multiple resonances' achievement, high reliability and reduced costs recommend fractal antennas for a series of applications ${ }^{(5-8)}$. Fractal antennas development recommend today for their use in ultra- and super-high frequency bands. Among them, the Sierpinski fractal proved its ability to solve interesting applications ${ }^{(9-14)}$ and may also be used for remote vital signs sensing.

Continuous-wave (CW) Doppler radar can sense the minute movements of the human body. Vital signs, respectively respiration and heartbeat, can be quantified based on the periodic shift in the phase of the transmission coefficient $S_{21}$ when the electromagnetic wave impinges the body surface. A series of applications were developed on this basis ${ }^{(15-17)}$ with the newest ones being able to quantify also the time-variability of the vital signs rhythms ${ }^{(18)}$. One of the essential components of such systems is the antenna (both the transmitting and the receiving ones). The problem is that low directivity antennas could conduct to significant pickup of the clutter and noise. Narrower bandwidth antennas may achieve a better signal-tonoise-plus-interference ratio performance than larger bandwidth antennas ${ }^{(19)}$. It has been demonstrated in ${ }^{(19)}$ that a high directivity antenna can indeed significantly improve the signal-to-noise-plus-interference ratio and robustness, thus providing better accuracy in recording the phase versus time variations. Therefore, in-house preparation of the antennas allows proprietary improvements when the system has to shift into detections of lives behind materials with various dielectric parameters and shielding properties or behind fire (disaster support). In this regard, the present work represents a part of a project devoted to the fabrication of PCB antennas for CW Doppler radar applications.

Since characterizing antennas by measurements is very resource-consuming, the modelling-simulation is the first step in featuring the approximate antenna parameters. Several commercial software is currently available. In this work, we used CST Studio Suite - 3D Electromagnetic Field Simulation Software. Then, a validation of the model antenna was made, by using standardized measurements in an accredited anechoic room. For the frequencies proving the highest total efficiency of the antennas, and at their known and largest gain, a raw set-up containing only the antennas and a vector network analyzer were used, to check the capability of determining the respiration frequency and the heart rate (pulse) by simply applying FFT processing of the time-domain recorded data.

\section{Materials and Methods}

Two antenna models were chosen for the present project: a microstrip patch and a triangular Sierpinski fractal (Figure 1 ). The models were designed using CST Studio Suite Software. The conducting surfaces of the antennas are made of copper with a width of $0.035 \mathrm{~mm}$ while the dielectric substrate is made of FR4 material having a height of $1.5 \mathrm{~mm}$. Dielectric properties of the substrate have a significant impact on the simulation results, and since they vary by manufacturer and from lot-to-lot, we had to choose a set of values not necessarily exactly the same as the ones of the real material we used in the experiments. We made computations considering dispersion laws for both the dielectric constant $\varepsilon_{r^{\prime}}$ and the tangent loss, $\varepsilon_{r^{\prime \prime}} / \varepsilon_{r^{\prime}}$. For the patch antenna, $\varepsilon_{r^{\prime}}$ varied in the range $(4.25-4.30)$ and $\varepsilon_{r^{\prime \prime}} / \varepsilon_{r^{\prime}}$ in the range $(0.016-0.018)$ for the frequency band of interest. For the Sierpinski fractal antenna, $\varepsilon_{r^{\prime}}$ varied in the range (4.30-4.31) while $\varepsilon_{r^{\prime \prime}} / \varepsilon_{r^{\prime}}$ in the (0.140-0.142) in the frequency band of interest. The FR4 dielectric data were extracted from ${ }^{(20)}$. 


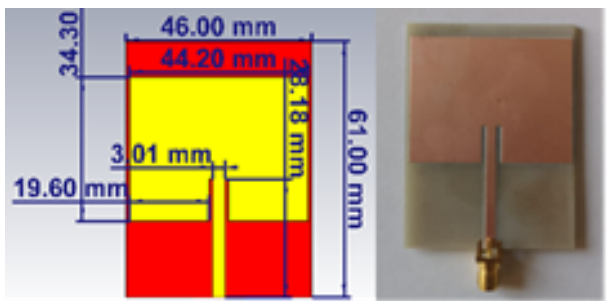

a

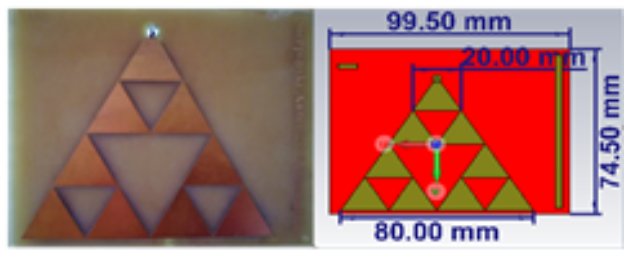

b

Fig 1. Models of the antennas and their dimensions, together with the pictures of the real, fabricated ones: a) microstrip patch antenna; b) Sierpinski fractal antenna.

At the patch antenna, the signal is applied practically through an SMA connector using a microstrip line with inset feeding while the simulation is applied by a waveguide port. The Sierpinski fractal is fed through a BNC connector experimentally, while in simulation a BNC connector was simulated using a waveguide port.

The real antennas were fabricated by using an NVIS 72 prototype machine from NVIS Technologies Pvt. Ltd. (Figure 2 ). The antenna models designed in CST Studio were exported as Gerber files and converted into G files using FlatCAM. After that, the files were imported into the NVIS-72 application connected to the prototyping machine.

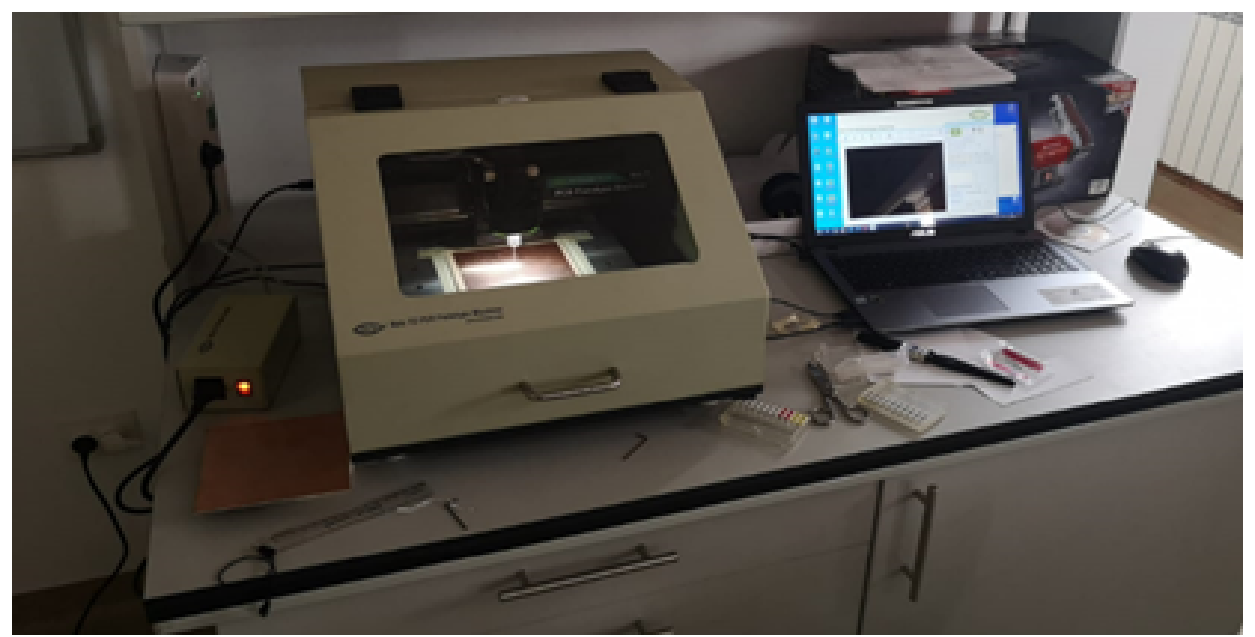

Fig 2. Fabricating the antennas with the NVIS 72 PCB prototyping machine.

The antennas were characterized both by simulation and by measurements in far-field conditions. Simulations were validated by measurements made in a shielded anechoic room in which a log-periodic antenna model USLP 9143 from Schwarzbeck was used at the receiver. The followed procedure is indicated in ${ }^{(21)}$. $3 \mathrm{D}$ radiation pattern together with polar plots of the gain at azimuth and elevation angles of interest, standing wave ratio (SWR) and the total efficiency were measured. In $^{(22)}$ an alternative experimental method of calculating the total efficiency is described, but the present experimental set-up allowed the direct determination of the total efficiency, containing both the radiation efficiency and mismatch losses. Comparisons between simulated and measured values have been performed. The frequency ranges of interest were: a) for the patch antenna, (2-5) GHz; b) for the Sierpinski fractal antenna, (8-9) GHz.

The principle by which the CW Doppler radar functions to detect breathing and heart beating, therefore chest position changes is based on the relationship between the signal's phase variation, $\Delta \theta(\mathrm{t})$, and the chest displacement, $\Delta \mathrm{x}(\mathrm{t})$ :

$$
\triangle \theta(t)=\frac{4 \pi}{\lambda} * \triangle x(t)
$$

where $\lambda$ is the wavelength that is propagating between the antenna and the target body. The incident wave, upon reflection from the chest, will contain information about respiration and the heartbeat when a person is breathing normally. The average peakto-peak chest displacement due to respiration is (4-12) $\mathrm{mm}$, and due to the heartbeat is $(0.2-0.5 \mathrm{~mm})$. At rest, the respiration 
frequency varies in the (0.1-0.3) $\mathrm{Hz}$ range and the pulse in the (1-3) Hz range ${ }^{(16)}$. Considering relationship (1), phase excursion of the transmission coefficient $S_{21}$ is expected to be much higher at larger frequencies. Accuracy detection of vital signs depend however on antenna parameters also ${ }^{(19)}$ and the most important are the gain and the bandwidth.

The measurements of the phase of the scattering parameter $S_{21}$ were made by a vector network analyzer model $\mathrm{ZNB} 40$ from Rohde \& Schwarz. The measurement calibration has always been rigorously made before each set of measurements by using the ZV-Z229 calibration kit and the standard methodology. The power input in the emitting antennas was always set at $8 \mathrm{dBm}$ at the vector network analyzer. The phase variation was recorded at the receiving antennas by setting the next values at the vector network analyzer: several points $=20.000$, recording duration $=30 \mathrm{~s}$, therefore the sampling frequency $=0.66 \mathrm{kHz}$. To validate the recording system, the heartbeat was simultaneously counted by a precision pulse-oximeter. The distance between the person's chest and the antenna plane was $40 \mathrm{~cm}$.

A parameter not usually mentioned in the literature is the incident power density impinging the chest of the person whose vital signs detection by the Doppler radar technique is done. We can estimate the average incident power density (PD) based on the formula:

$$
P D=\frac{G * P}{4 \pi * d^{2}}
$$

where $\mathrm{G}$ is the averaged numeric gain of the emitting antenna over the angle of incidence to the chest, $\mathrm{P}$ is the effective emitted power of the antenna (taking into consideration its total efficiency) and $d$ is the distance between the antenna surface and the chest surface. A more precise method is however the direct measurement of the local PD and then its spatial averaging over the chest surface. Therefore, in the present work we have measured it by using a signal generator model Apsin $20 \mathrm{G}$ up to $20 \mathrm{GHz}$ from Ana Pico which fed $8 \mathrm{dBm}$ power into the antennas, a real-time spectrum analyzer model Spectran HF 80120 V5-X from Aaronia to measure the received power and a miniature E-field probe model PBS-1 from Aaronia, which allows determination of the measured E-field strength in the air, by using specific converting files. Finally, the local PD was determined by the relation (far-field):

$$
P D=\frac{E^{2}}{Z_{0}}
$$

where $Z_{0}$ is the free space impedance.

\section{Results and Discussion}

\subsection{Characteristics of the proposed antennas}

The total efficiency of the antenna is determined by the summed effects of impedance mismatches and radiation losses. Figure 3 shows the variation of the total efficiency of the antennas in the frequency ranges of interest. It can be observed that measured values of efficiency are always higher than the simulated ones. The patch antenna shows at $\mathrm{f}=2.12 \mathrm{GHz}$ an experimental efficiency of $43 \%$, while the Sierpinski fractal shows at $f=(8.8-8.9) \mathrm{GHz}$ an efficiency of $65 \%$. These values are the highest obtained, so the frequencies chosen for the Doppler radar measurements will be situated at these values.

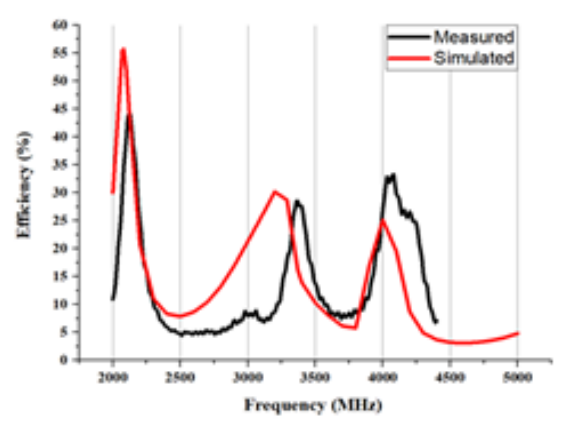

a

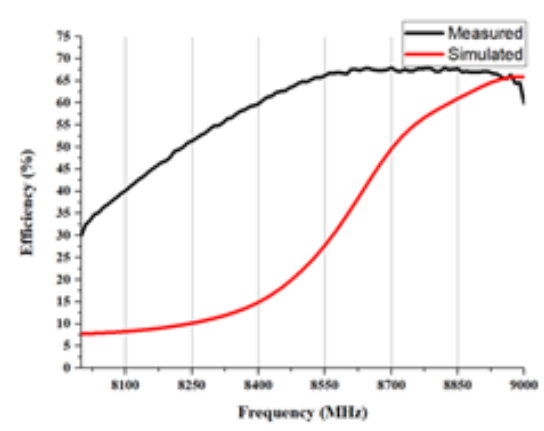

b

Fig 3. Total efficiency of the antennas, measured values versus simulated ones: a) microstrip patch antenna; b) Sierpinski fractal antenna. 
SWR variation with frequency, which is an indicator of the reflected power ratio, is presented in Figure 4 . In this case, again, better results were obtained in the measurements than in the simulations, and they were considered in the assessments. Two narrow resonance frequency bands and a larger one are identified on the SWR graph of the patch antenna, while multiple resonance narrow bands and an ultra-wideband one are observed at the Sierpinski antenna. The patch antenna has a bandwidth of $11.4 \mathrm{MHz}$ and the main resonance at $2.12 \mathrm{GHz}$. The large bandwidth of the Sierpinski antenna at the resonance of $8.82 \mathrm{GHz}$ is $317.2 \mathrm{MHz}$, which recommends it also for applications of very high-speed data communications - ultra-wideband. Figure 4 contains also magnifications of the zones containing minimums of SWR to observe with enhanced accuracy the measured and simulated values.

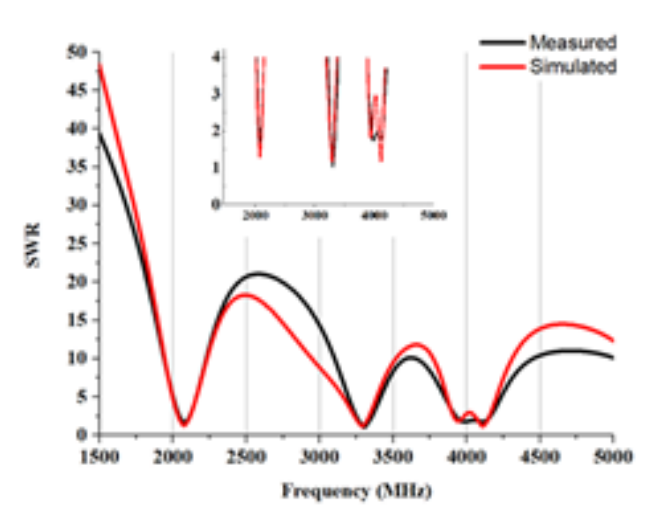

a

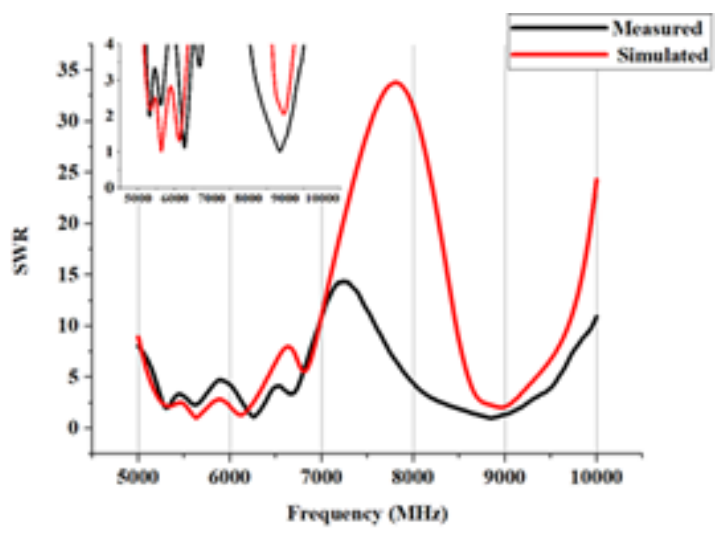

b

Fig 4. Stationary Wave Ratio over the investigated frequency range, measured values versus simulated ones; magnifications of resonances are outlined in the upper part of the figures: a) microstrip patch antenna; b) Sierpinski fractal antenna.

Figure 5 emphasizes the 3D radiation patterns of the two antennas at the frequencies of interest. Neither of them is very directive. The principal radiation direction is practically perpendicular to the active surface of the patch antenna and the maximum gain is $3.15 \mathrm{dBi}$. Sierpinski antenna has almost an omnidirectional behavior, but there are some narrow angles at which the gain decreases drastically. The principal radiation direction is situated almost parallel to the active surface of the antenna, in the direction of the large triangle basis and its maximum gain is $5.5 \mathrm{dBi}$.

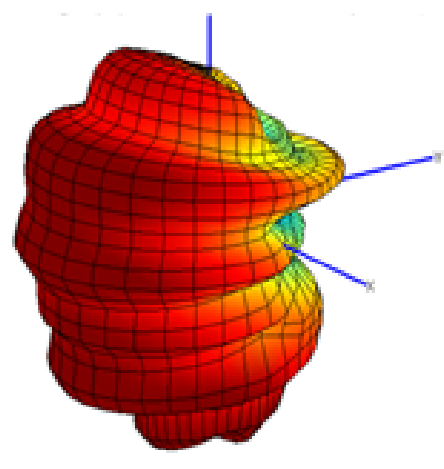

a

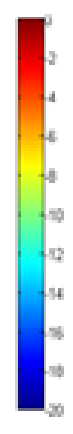

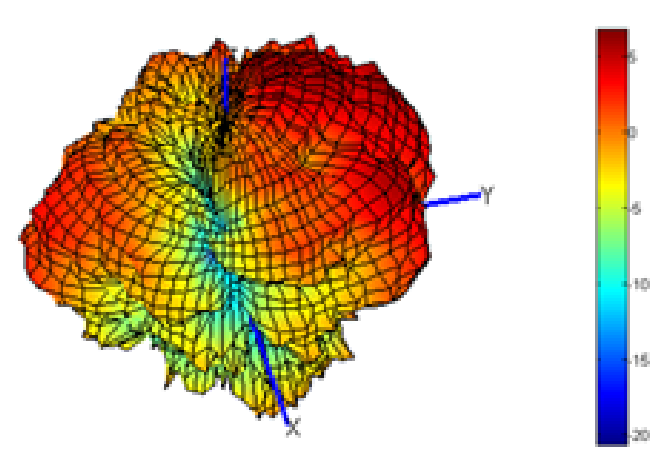

b

Fig 5. Measured radiation patterns of the antennas and their gains in $\mathrm{dB}$ in the legends: a) microstrip patch antenna at $2.12 \mathrm{GHz}$; $\mathrm{PCB}$ plane is $\mathrm{xOz}$; b) Sierpinski fractal antenna at $8.9 \mathrm{GHz}$; $\mathrm{PCB}$ plane is $\mathrm{xOy}$. 
A piramidal volumetric Sierpinski fractal antenna was proposed in ${ }^{(23)}$ using the dielectric Rogers-RT/Duroid 6010, which is a much expensive material than FR4. The expense of that dielectric and the larger spatial span provided a much larger bandwidth, a maximum gain $1 \mathrm{~dB}$ higher and an efficiency $16 \%$ larger than the present planar Sierpinski antenna.

Comparison between measurements and simulations of radiation patterns of the patch and Sierpinski antennas are observed in Figures 6 and 7 in which the representations of polar plots are given. They show comparatively the gains of co-polar components, cross-polar components and respectively the total values. The situations in Figure 6 correspond to the patch antenna, while those in Figure 7 correspond to Sierpinski antenna, at resonance frequencies. The graphs in both figures correspond to the situations: a) first raw presents the measured values; b) second raw presents the simulated values; $c$ ) left side graphs correspond to azimuth cut at $\theta=90 \mathrm{deg}$; d) right side graphs correspond to elevation cut at $\varphi=0 \mathrm{deg}$. The representations in these figures sustain earlier findings ${ }^{(24)}$ which showed that the substrate permittivity and thickness are responsible for both the bandwidth and the radiated cross-polar level. In case of thick and low permittivity dielectric which enlarges the bandwidth, the radiation pattern becomes affected by a high cross-polar level. Thinner substrate and medium permittivity conduct to a lower cross-polar level, but to a narrower bandwidth.
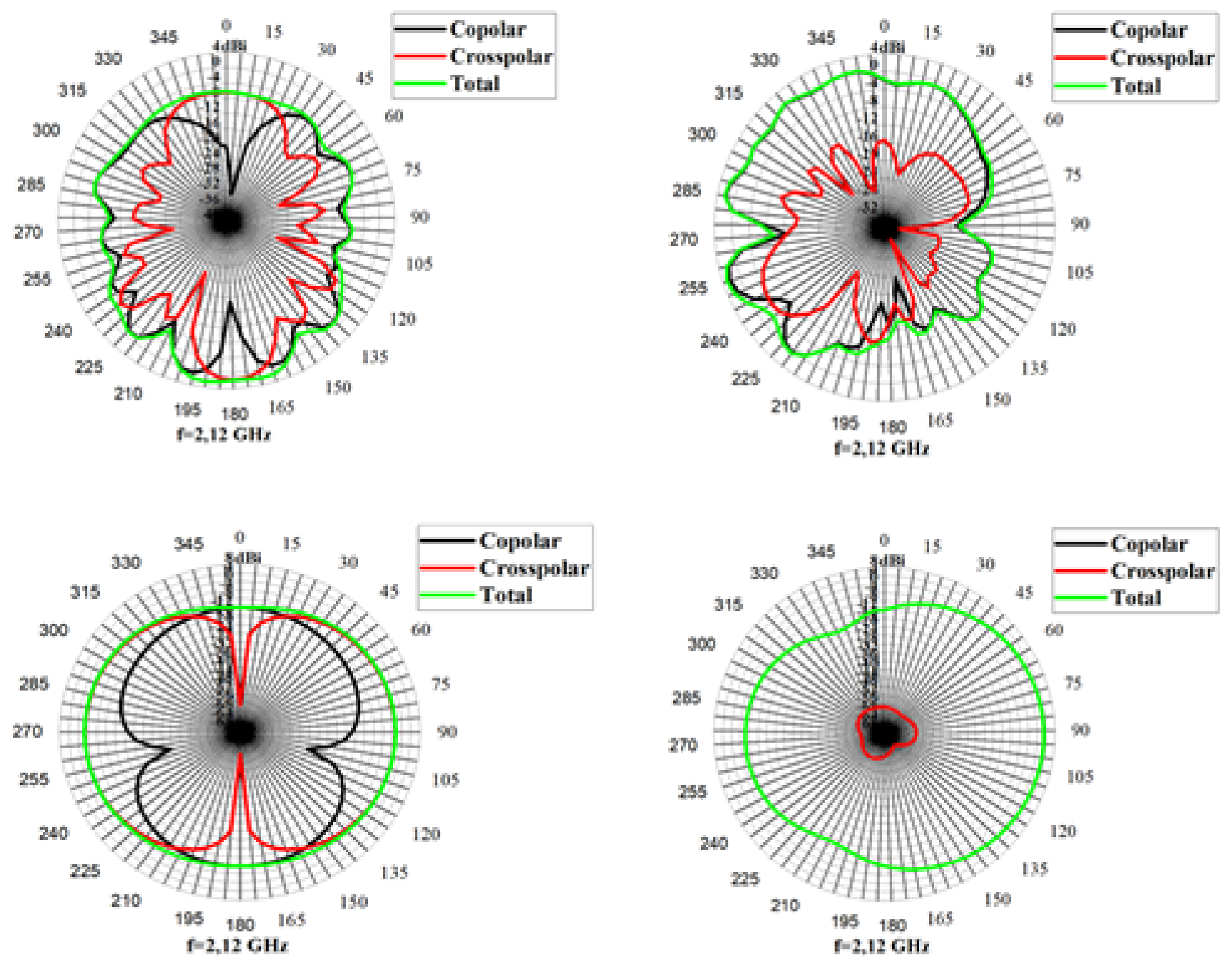

Fig 6. Polar plots of the gains for the microstrip patch antenna at $2.12 \mathrm{GHz}$ : measured (first row) versus simulated (second r0w); left sides azimuth, theta $=90 \mathrm{deg}$; right sides - elevation, $\mathrm{phi}=90 \mathrm{deg}$. 

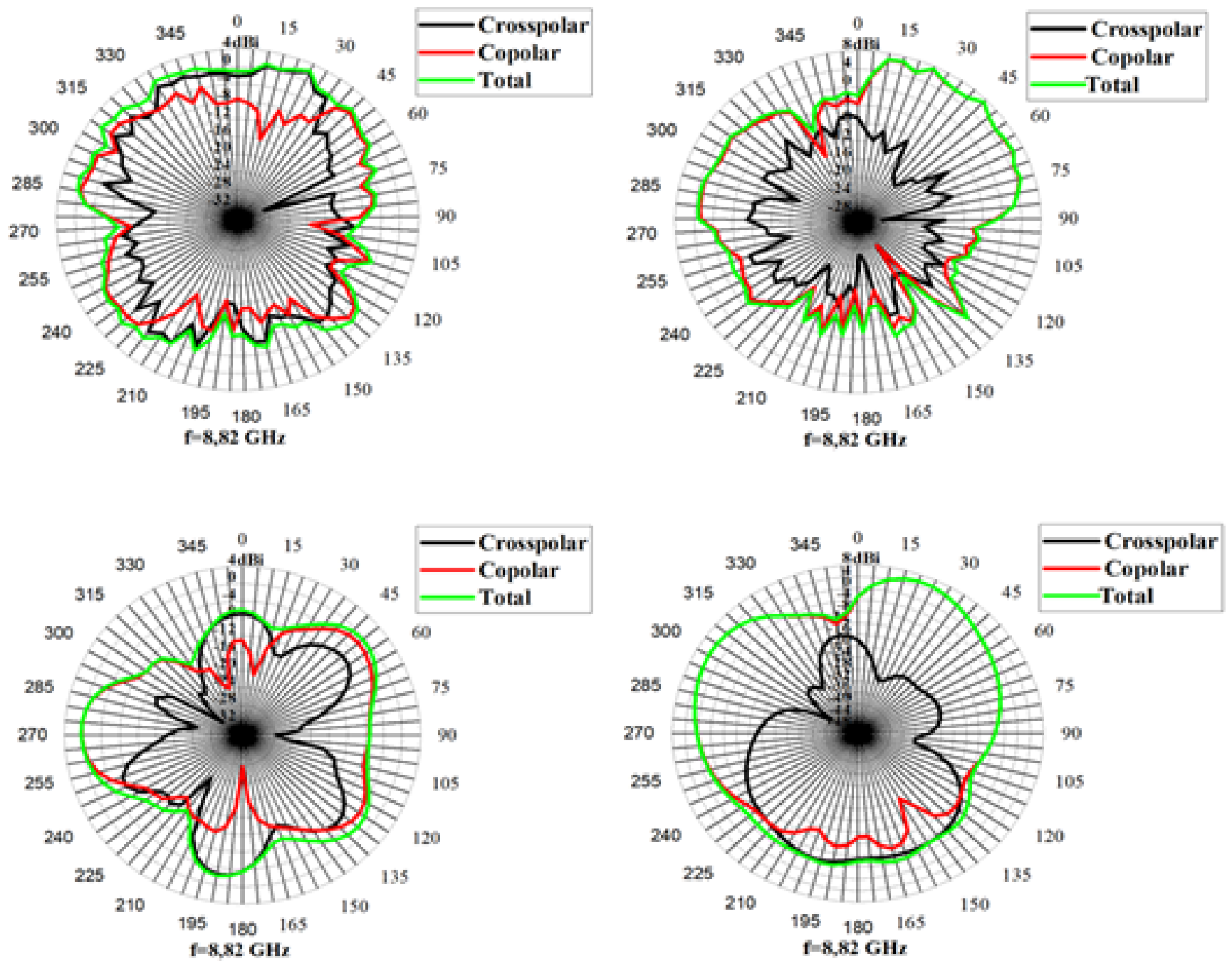

Fig 7. Polar plots of the gains for the Sierpinski fractal antenna at $8.82 \mathrm{GHz}$ : measured (first row) versus simulated (second row); left sides azimuth, theta $=90 \mathrm{deg}$; right sides - elevation, phi $=90 \mathrm{deg}$.

\subsection{Breath and pulse detection with the antennas}

As indicated in relation (1) the variation of the $S_{21}$ phase due to chest movements is larger for higher frequencies. Therefore, increased sensitivity to minute movements at higher frequencies may overpower the noise ${ }^{(24)}$. When the used antenna shows higher total efficiency, higher gain and narrower radiation lobes that impact the chest of the person, the overall result is also the diminishing of the noise from the surrounding environment. Frequency gradual increase encounters however a phenomenological threshold due to the enlarging of the negative effects of the harmonic interference and inter-modulation interference, which will conduct to a decrease in the detection accuracy. This is mainly the case when the harmonics of the breathing rate are close to the heart rate. The accuracy is also more negatively affected in the case when the heart rate and the breathing rate are very close. Literature shows that heart rate can however be accurately detectable by frequencies up to $26 \mathrm{GHz}$ with improved systems ${ }^{(25)}$. The extra noise due to the coaxial cables and connectors had another effect on vital signs detection accuracy, especially at higher frequencies. Also, at larger frequencies $(>9 \mathrm{GHz})$, numerous artefacts may affect the measurement accuracy, for example, unwanted motions, non-uniform chest motion-captured due to non-uniform illumination by the incident wave, etc. All of these conduct to the apparition of numerous spikes that conduct to confounding the useful signal. Advanced signal processing techniques involving harmonic cancellation can lead to improved accuracy.

In the case of our experiments, the unfiltered signals from the vector network analyzer were used for frequency spectrum generation. In Figure 8 we observe the disposal of the antennas about the person's chest during the vital signs recordings at both 
frequencies. On the finger, the person wears the pulse-oximeter, as a reference of the heart rate determination. Breathing was referenced based on the own time-counting of the number of respirations and their total duration.

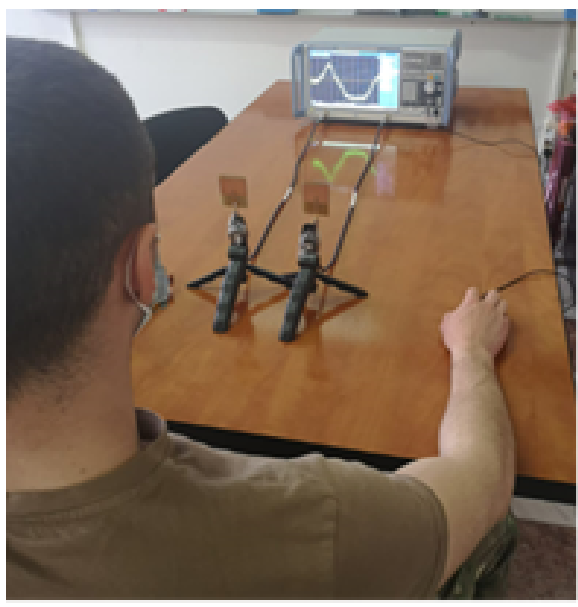

a

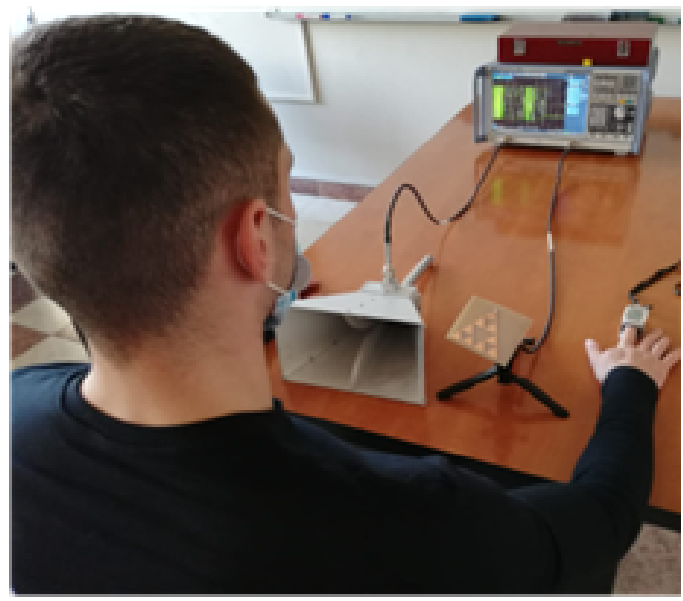

b

Fig 8. Experimental set-up used for recording the breath and heartbeats of a person with the antennas connected to the vector network analyzer: a) microstrip patch antenna as transmitter and receiver; b) horn antenna as transmitter and Sierpinski fractal antenna as receiver.

Using the miniature E-field probe, we determined the following average values of the incident power density impinging the chest of the person: $\mathrm{PD}_{\text {patch }}=4.5 \times 10^{-4} \mathrm{~mW} / \mathrm{m}^{2}$ at $2.12 \mathrm{GHz} ; \mathrm{PD}_{\text {horn }}=2.6 \times 10^{-2} \mathrm{~mW} / \mathrm{m}^{2}$ at $8.82 \mathrm{GHz}$ (the horn antenna was the transmitting one and the Sierpinski antenna was the receiving one).

In Figure 9 a set of five traces of the respiration are represented evolving in time, during the recording duration of $30 \mathrm{~s}$ at 2.12 $\mathrm{GHz}$ using the patch antenna. FFT spectrum of one of them is presented on the lateral side of the figure. The raw spectrum results in a breathing frequency of $0.13 \mathrm{~Hz}$ and a heartbeat frequency of $1.56 \mathrm{~Hz}$. Both frequencies correspond with the averaged ones measured directly, therefore a very good accuracy was gained. It resulted that the magnitude of the heart rate is approximately 8 times lower than that of respiration. The alternative measurements showed average values of $0.16 \mathrm{~Hz}$ for breathing and 1.60 $\mathrm{Hz}$ for heartbeat frequency.

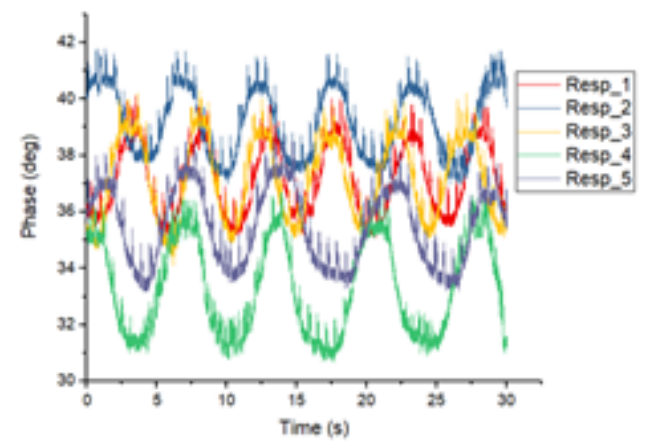

a

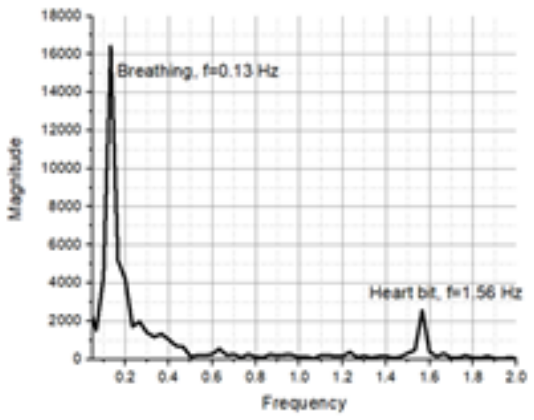

b

Fig 9. a) Phase variation of $S_{21}$ transmission parameter during 30 seconds of vital signs recordings (five repetitions) with the patch-patch antennas on $2.12 \mathrm{GHz}$; b) FFT spectrum of one of the recordings emphasizing the frequencies of the respiration and of the heartbeat.

In Figure 10 a set of five traces of the respiration recorded with the Sierpinski antenna during $30 \mathrm{~s}$ is presented. At a higher frequency, higher phase variation is observed, as predicted by equation (1). The FFT spectrum of one of the time representations is shown in lateral. It is observed that the breathing frequency was $0.16 \mathrm{~Hz}$ and the pulse frequency was $1.37 \mathrm{~Hz}$. The alternative 
measurements showed average values of $0.18 \mathrm{~Hz}$ for breathing and $1.40 \mathrm{~Hz}$ for heartbeat frequency. At $8.82 \mathrm{GHz}$, with the horn-Sierpinski antennas, the magnitude of the heart rate is approximately 23 times lower than that of respiration. The vital signs frequencies have been correctly extracted, as compared with the direct measurements, but the presented spectrum is one of the best ones because generally many spikes of approximately the same magnitude could be observed at different frequencies, illustrating the presence of phenomena of artefacts and interferences commented above. In this case, without having a reference to identify the heartbeat frequency, it would be practically impossible to distinguish the heart rate signal.

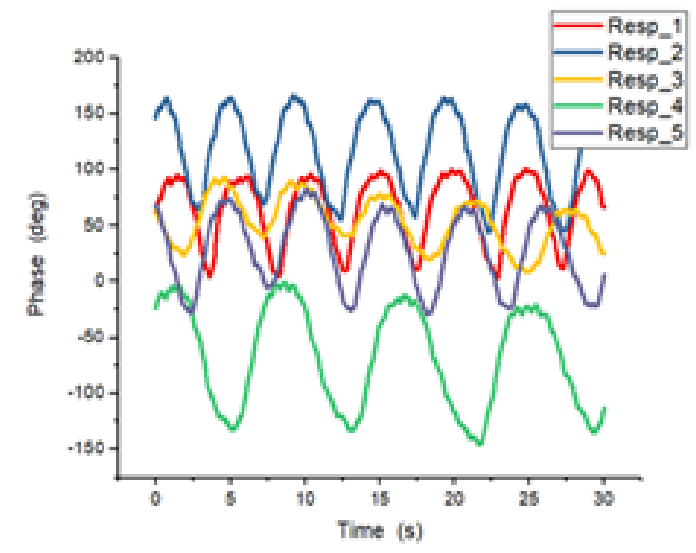

a

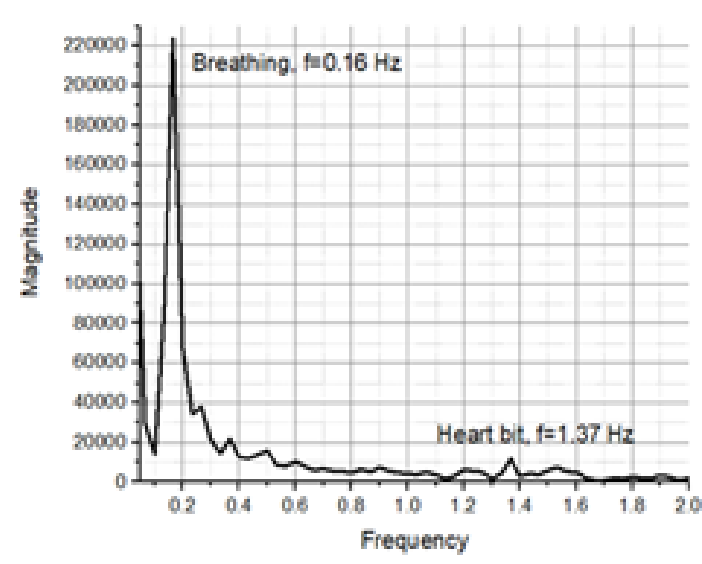

$\mathrm{b}$

Fig 10. a) Phase variation of $S_{21}$ parameter during 30 seconds of vital signs recordings (five repetitions) with the Horn-Sierpinski fractal antennas on $8.82 \mathrm{GHz}$; b) FFT spectrum of one of the recordings emphasizing the frequencies of the respiration and of the heartbeat.

Figure 11 shows a comparative magnification of the phase-time recordings at the two different frequencies. It is interesting to note that the heart rate may be identified with the naked eye in the graphs: the small spikes distributed along the larger phase excursions corresponding to respiration are due to the heart rhythm. The phase shift due to the heartbeats is of about 1 degree at $2.12 \mathrm{GHz}$ as captured by the patch antenna and of about 6 degrees at $8.82 \mathrm{GHz}$ as captured with the Sierpinski antenna. These time-series data enabled very good results for the frequency spectrum representations using the classical FFT transformation while applying no hardware or software improvements to the raw data. The used frequencies do not have any significant effect on the accuracy of respiration rhythm identification. At a higher frequency, the decreased accuracy of heart rate determination is due to the presence of increased harmonic and intermodulation interferences. Generally, in these experiments both antennas and frequencies could detect both vital signs directly, paving the way for further developing the system based on these antennas.

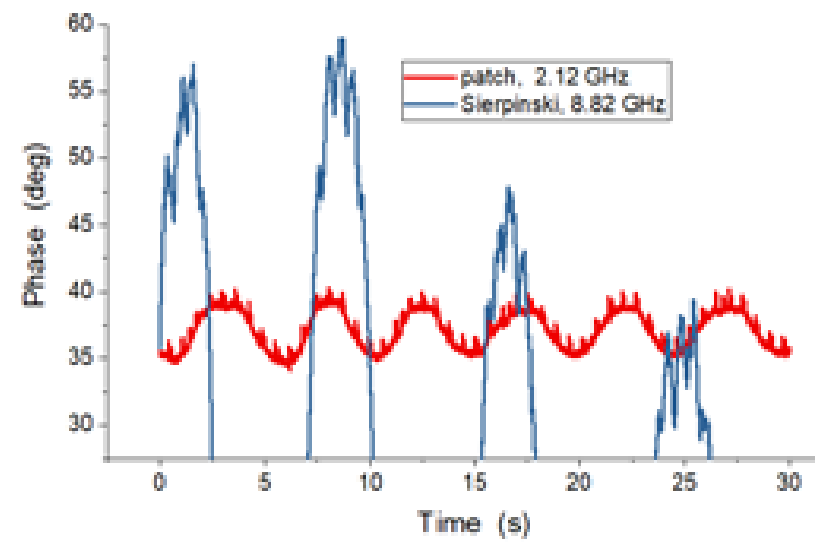

Fig 11. Details of time-representation of vital signs: observing the phase modifications on the same scale due to heartbeat which are the small oscillations inside the larger ones that correspond to breathing 
Literature shows that generally the detection of the heartbeat became impossible without consecrated detection system architectures based on quadrature reception. Such systems also needed compensation of the imperfections between the two quadrature signals because a direct current offset and additional reflections due to clutter are always present at the receiver ${ }^{(15)}$. A set of solutions to compensate these undesirable effects exist ${ }^{(26)}$, and for example the baseband signal processing may be approached by adaptive algorithms such as Multiple Signal Classification parametric spectral estimation, that provided an accurate identification of the heart rate even after short duration measurements ${ }^{(15)}$. With the present approach, of careful characterization of the antennas, part of these problems could be surpassed.

\section{Conclusion}

A triple-band rectangular patch antenna was designed and characterized both by simulation and by measurements for further applications. One of the resonance frequencies presented the highest total efficiency of $43 \%$ at $2.12 \mathrm{GHz}$ where the gain was $3.15 \mathrm{dBi}$ while the main radiation lobe was very large. This frequency was considered proper for a Doppler radar application following the detection of both the respiration and the heartbeat of a person standing in front of the antenna with no interposed obstacles.

A second planar antenna, a Sierpinski triangle fractal, was also designed and characterized and showed an ultra-wideband profile centered on $8.82 \mathrm{GHz}$ with a bandwidth of $317 \mathrm{MHz}$. With a total efficiency of $65 \%$ at this resonant frequency, with a gain of $5.55 \mathrm{dBi}$ and with a very large main radiation lobe, it was also considered proper for the radar detection of vital signs.

By using the simplest CW Doppler radar system constituted by only these antennas and a vector network analyzer, with no inserted filters or amplifiers and by applying the classical FFT processing, we were able to extract the respiration and the heartbeat frequencies of a person situated at $40 \mathrm{~cm}$ distance from the antennas. At the higher frequency however, some limitations are observed: due to unwanted movements and unstable environment, due to harmonic interference and intermodulation interference, recording a single trace of phase variation during $30 \mathrm{~s}$ long will not be sufficient to correctly extract the heartbeat frequency. It is advised to repeat recording a few times, to extract the signal without supplementary processing. The Doppler radar system containing either of the antenna models can be further developed for sensing the vital signs when obstacles will be present in the path of the incident and reflected waves or when the subject will be situated behind the fire.

\section{Acknowledgement}

We address special thanks for the initial scientific support offered in developing fractal antennas to Professor Marie Richterova and Mr. Miroslav Popela from the University of Defence - Faculty of Military Technology, Brno, Czech Republic.

\section{References}

1) Bankey V, Kumar NA. Design and performance issues of Microstrip antennas. International Journal of Scientific and Engineering Research. 2015;6(3):15721580. Available from: https://dx.doi.org/10.14299/ijser.2015.03.008.

2) Liu Y, Si LM, Wei M, Yan P, Yang P, Lu H, et al. Some recent developments of microstrip antenna. International Journal of Antennas and Propagation. 2012;(428284). Available from: https://doi.org/10.1155/2012/428284.

3) Minhas S, Khosla D, and. Compact Size and Slotted Patch Antenna for WiMAX and WLAN. Indian Journal of Science and Technology. 2017;10(16):1-5. Available from: https://dx.doi.org/10.17485/ijst/2017/v10i16/102762.

4) Singh A, Bera R, Maji B, Gurung R. Microstrip Patch Antenna towards Future of Communication. Indian Journal of Science and Technology. 2019;12(3):15. Available from: 10.17485/ijst/2019/v12i3/140904.

5) Anguera J, Andújar A, Jayasinghe J, Chakravarthy VVSSS, Chowdary PSR, Pijoan JL, et al. Fractal Antennas: An Historical Perspective. Fractal and Fractional. 2020;4(1):3. Available from: https://dx.doi.org/10.3390/fractalfract4010003.

6) Sultan QH, Sabaawi AMA. DESIGN AND IMPLEMENTATION OF IMPROVED FRACTAL LOOP ANTENNAS FOR PASSIVE UHF RFID TAGS BASED ON EXPANDING THE ENCLOSED AREA. Progress In Electromagnetics Research C. 2021;111:135-145. Available from: https://dx.doi.org/10. 2528/pierc21012206.

7) Salama AMA, Quboa KM. Fractal dipoles as meander line antennas for passive UHF RFID tags. 5th International Multi-Conference on Systems, Signals and Devices. 2008;p. 1-6. Available from: 10.1109/SSD.2008.4632843.

8) Sabaawi AMA, Quboa KM. Design and Fabrication of Miniaturized Fractal Antennas for Passive UHF RFID Tags. In: Preradovic S, et al., editors. Advanced Radio Frequency Identification Design and Applications. InTech. 2011;p. 29-50. Available from: 10.5772/14372.

9) Gupta S, Kshirsagar P, Mukherjee B. Sierpinski fractal inspired inverted pyramidal DRA for wide band applications. Electromagnetics. 2018;38(2):103-112. Available from: https://dx.doi.org/10.1080/02726343.2018.1436738.

10) Jayarenjini N, Unni C. MERR Inspired CPW Fed SSGF Antenna for Multiband Operations. Progress In Electromagnetics Research C. $2019 ; 91: 197-211$. Available from: https://dx.doi.org/10.2528/pierc19020202.

11) Akhtar N, Bhomia Y, Bhardwaj A, , and. Design and Simulation of Sierpinski Carpet Antenna utilizing Two Feeding Method. Indian Journal of Science and Technology. 2017;10(35):1-7. Available from: https://dx.doi.org/10.17485/ijst/2017/v10i35/118957.

12) Gupta M, Mathur V. SierpinskiArray with Swastik Electromagnetic Bandgap for Ku-Band Applications. Indian Journal of Science and Technology. 2016;9(32):1-7. Available from: https://dx.doi.org/10.17485/ijst/2016/v9i32/93833. 
13) Vatamanu D, Miclăuş S. UHF Fractal Antennas: Solutions for Radio Links Using Matlab Simulations. International conference KNOWLEDGE-BASED ORGANIZATION. 2020;26(3):179-184. Available from: https://dx.doi.org/10.2478/kbo-2020-0135.

14) Vatamanu D, Miclaus S. An analysis of the Influence of the Dielectric Substrate Parameters on the Performances of Koch and Minkowski Single-Iteration Fractal Antennas. European Journal of Advances in Engineering and Technology. 2020;8(7):1-8.

15) Bechet $P$, Mitran R, Munteanu M. A non-contact method based on multiple signal classification algorithm to reduce the measurement time for accurately heart rate detection. Review of Scientific Instruments. 2013;84(8). Available from: https://dx.doi.org/10.1063/1.4818974.

16) El-Samad S, Obeid D, Zaharia G, Sadek S. Ghais El Zein. Measurements of Cardiac and Cardiopulmonary Activities Using Contactless Doppler Radar. International Conference on advances in Biomedical Engineering. 2015;p. 193-196. Available from: 10.1109/ICABME.2015.7323285.

17) Wang Y, Liu Q, Fathy AE. CW and Pulse-Doppler Radar Processing Based on FPGA for Human Sensing Applications. IEEE Transactions on Geoscience and Remote Sensing. 2012;51(5):1-11. Available from: https://dx.doi.org/10.1109/tgrs.2012.2217975.

18) Petrovic VL, Jankovic MM, Lupsic AV, Mihajlovic VR, Popovic-Bozovic JS. High-Accuracy Real-Time Monitoring of Heart Rate Variability Using 24 GHz Continuous-Wave Doppler Radar. IEEE Access. 2019;7:74721-74733. Available from: https://dx.doi.org/10.1109/access.2019.2921240.

19) Das V, Boothby A, Hwang R, Nguyen T, Lopez J, Lie DYC. Antenna evaluation of a non-contact vital signs sensor for continuous heart and respiration rate monitoring. In: IEEE Topical Conference on Biomedical Wireless Technologies, Networks, and Sensing Systems (BioWireleSS). 2012;p. 13-16. doi:10.1109/BioWireless.2012.6172730.

20) Paleček J, Vestenický M, Vestenický P, Spalek J. Frequency Dependence Examination of PCB Material FR4 Relative Permittivity. Elsevier BV. 2013. Available from: https://dx.doi.org/10.3182/20130925-3-cz-3023.00020. doi:10.3182/20130925-3-cz-3023.00020.

21) ANSI/IEEE Std 149-1979. IEEE Standard Test Procedures for Antennas. 1979. Available from: 10.1109/IEEESTD.1979.120310.

22) Mukherjee B, Patel P, Mukherjee J. Hemispherical Dielectric Resonator Antenna Based on Apollonian Gasket of Circles-A Fractal Approach. IEEE Transactions on Antennas and Propagation. 2014;62(1):40-47. Available from: https://dx.doi.org/10.1109/tap.2013.2287011.

23) Gupta S, Kshirsagar P, Mukherjee B. Sierpinski fractal inspired inverted pyramidal DRA for wide band applications. Electromagnetics. 2018;38(2):103-112. Available from: https://dx.doi.org/10.1080/02726343.2018.1436738.

24) García-García Q. Radiated cross-polar levels and mutual coupling in patch radiators. International Journal of RF and Microwave Computer-Aided Engineering. 2000;10(6):342-352. Available from: 10.1002/1099-047X(200011)10:6<342::AID-MMCE3>3.0.CO;2-0.

25) Tariq A. Vital Signs Monitoring using Doppler Radar and On-Body Antennas. 2012. Available from: https://etheses.bham.ac.uk/id/eprint/4332/1/ Tariq13PhD.pdf.

26) Llamas-Garro I, Melo MTD, Kim JM. Frequency Measurement Technology. 2018;p. 1-222. 